\title{
Bladder Urachal Adenocarcinoma
}

National Cancer Institute

\section{Source}

National Cancer Institute. Bladder Urachal Adenocarcinoma. NCI Thesaurus. Code C39843.

A rare bladder adenocarcinoma that develops from the urachal remnant. 